\title{
REACTION-DIFFUSION SYSTEMS WITH SKEW-GRADIENT STRUCTURE
}

\author{
EIJI YANAGIDA*
}

\begin{abstract}
The aim of this paper is to introduce reaction-diffusion systems with skew-gradient structure and review recent studies on the stability of steady states of the skew-gradient systems. The reaction-diffusion system with skew-gradient structure is a sort of activator-inhibitor system that consists of two gradient systems coupled in a skew-symmetric way. Due to such structure, the system enjoys some nice mathematical properties that help us to study the behavior of solutions analytically. First, we consider standing pulse solutions on $\mathbf{R}$, and give a simple criterion for the instability of the standing pulse. Second we give a general criterion for the Eckhaus instability of spatially periodic steady states on $\mathbf{R}$. Finally, in the case of bounded domains, we show a relation between the stability properties of the steady state and mini-maximizing properties for some functional.
\end{abstract}

1. Introduction. It is known that various interesting spatial patterns are observed in reaction-diffusion systems of activator-inhibitor type, and many mathematical results have been obtained concerning the behavior of solutions. However, since such systems are not order-preserving and the linearized operators around steady states are not self-adjoint, their mathematical analysis is extremely difficult and a general approach does not seem to be fruitful. Therefore, in order to study the existence and stability of large amplitude steady states, we usually introduce a small parameter to the system and consider a singularly perturbed problem.

In this paper, we adopt a different approach for the stability problem. Instead of introducing a small parameter, we introduce special structure called skew-gradient structure. The purpose of this paper is to review recent studies on the skew-gradient systems [5], [9], [10].

Let us consider $(m+n)$-component reaction-diffusion system

$$
\left\{\begin{array}{l}
S u_{t}=C \Delta u+f(u, v), \\
T v_{t}=D \Delta v+g(u, v),
\end{array}\right.
$$

where $u(x, t)={ }^{t}\left(u_{1}, \ldots, u_{m}\right)$ and $v(x, t)={ }^{t}\left(v_{1}, \ldots, v_{n}\right), S$ and $C$ are $m$ th order positive diagonal matrices, $T$ and $D$ are $n$th order positive diagonal matrices. We say that the system (1.1) has skew-gradient structure if for some $C^{3}$-function $H(u, v): \mathbf{R}^{m+n} \rightarrow \mathbf{R}$, the nonlinear terms $f={ }^{t}\left(f_{1}, \ldots, f_{m}\right): \mathbf{R}^{m+n} \rightarrow \mathbf{R}^{m}$ and $g={ }^{t}\left(g_{1}, \ldots, g_{n}\right): \mathbf{R}^{m+n} \rightarrow \mathbf{R}^{n}$ are expressed as

$$
f(u, v)=+\nabla_{u} H(u, v), \quad g(u, v)=-\nabla_{v} H(u, v),
$$

where $\nabla_{u}$ and $\nabla_{v}$ are gradient operators with respect to $u$ and $v$, respectively, i.e.,

$$
\nabla_{u}:={ }^{t}\left(\frac{\partial}{\partial u_{1}}, \ldots, \frac{\partial}{\partial u_{m}}\right), \quad \nabla_{v}:={ }^{t}\left(\frac{\partial}{\partial v_{1}}, \ldots, \frac{\partial}{\partial v_{n}}\right) .
$$

(More general definitions of skew-gradient structure are given in [5], [10].) Throughout this paper, we assume that (1.1) has skew-gradient structure.

${ }^{*}$ Mathematical Institute, Tôhoku University, Sendai 980-8578, Japan (yanagida@math.tohoku.ac.jp). 
We note that there are many examples of skew-gradient systems. An important example of the skew-gradient system is the FitzHugh-Nagumo system with diffusion:

$$
\left\{\begin{array}{l}
u_{t}=\Delta u+f(u)-v, \\
v_{t}=d \Delta v+\varepsilon(u-\gamma v) .
\end{array}\right.
$$

Indeed, by setting

$$
H(u, v):=\int f(u) d u-u v-\frac{1}{2} \gamma v^{2}
$$

(1.3) is rewritten as

$$
\left\{\begin{array}{c}
u_{t}=\Delta u+\frac{\partial H}{\partial u}, \\
\varepsilon^{-1} v_{t}=\varepsilon^{-1} d \Delta v-\frac{\partial H}{\partial v} .
\end{array}\right.
$$

Another important example is the Gierer-Meinhardt system

$$
\left\{\begin{aligned}
u_{t} & =\varepsilon^{2} \Delta u-u+\frac{u^{p}}{v^{q}}+\sigma \\
\tau v_{t} & =d \Delta v-v+\frac{u^{r}}{v^{s}}
\end{aligned}\right.
$$

which has skew-gradient structure when $p+1=r$ and $q+1=s$. Indeed, by setting

$$
H(u, v):=-\frac{r}{2} u^{2}+\frac{q}{2} v^{2}+\frac{u^{r}}{v^{q}}+r \sigma u,
$$

(1.4) is rewritten as

$$
\left\{\begin{array}{l}
r u_{t}=r \varepsilon^{2} \Delta u+\frac{\partial H}{\partial u} \\
q v_{t}=q d \Delta v-\frac{\partial H}{\partial v}
\end{array}\right.
$$

Notice that both of the above examples are of activator-inhibitor type. In general, any two-component reaction-diffusion system

$$
\left\{\begin{array}{l}
\tau_{1} u_{t}=d_{1} \Delta u+f(u, v), \\
\tau_{2} v_{t}=d_{2} \Delta v+g(u, v),
\end{array}\right.
$$

has skew-gradient structure if the nonlinearities satisfy

$$
\frac{\partial f}{\partial v}=-\frac{\partial g}{\partial u} \quad\left(=\frac{\partial H^{2}}{\partial u \partial v}\right) .
$$

Therefore, the skew-gradient system is neither a cooperation system nor a competition system so that it is not order-preserving. However, due to the skew-gradient structure, the system enjoys some nice mathematical properties that help us to study the behavior of solutions analytically.

In this paper, we discuss three problems and demonstrate how the skew-gradient structure is helpful for the analysis of reaction-diffusion systems. In Section 2, we 
consider stability problem associated with standing pulse solutions on $\mathbf{R}$, and give a simple criterion for the instability. In Section 3, we consider spatially periodic steady states on $\mathbf{R}$, and give a general criterion for the Eckhaus instability. Finally, in Section 4, we consider skew-gradient systems on bounded domains, and show relations between stability properties of steady states and mini-maximizing properties for some associated variational problem.

2. Standing Pulse Solutions. In this section, based on [9], we consider the skew-gradient system on $\mathbf{R}$ :

$$
\left\{\begin{array}{l}
S u_{t}=C u_{x x}+f(u, v), \\
T v_{t}=D v_{x x}+g(u, v),
\end{array} \quad x \in \mathbf{R} .\right.
$$

Any stationary solution $(u, v)=(u(x), v(x))$ of $(2.1)$ satisfies

$$
\left\{\begin{array}{l}
C u_{x x}+f(u, v)=0, \\
D v_{x x}+g(u, v)=0,
\end{array} \quad x \in \mathbf{R} .\right.
$$

Let $(p, q)$ be any critical point of $H(u, v)$. Then we have $f(p, q)=0$ and $g(p, q)=0$, which implies that $(u(x, t), v(x, t)) \equiv(p, q)$ is a spatially homogeneous stationary solution of (2.1). Assume that there exists a standing pulse solution $(u, v)=(\varphi(x), \psi(x))$. More precisely, $(\varphi(x), \psi(x))$ is a nonconstant function satisfying

$$
\left\{\begin{array}{l}
C \varphi_{x x}+f(\varphi, \psi)=0, \\
D \psi_{x x}+g(\varphi, \psi)=0, \\
(\varphi( \pm \infty), \psi( \pm \infty))=(p, q) .
\end{array} \quad x \in \mathbf{R}\right.
$$

In this section, we discuss stability of the standing pulse solution. We note that if the spatially homogeneous steady state $(u, v) \equiv(p, q)$ is not stable, then the standing pulse solution cannot be stable. Therefore, we assume in the following that the stationary solution $(u, v) \equiv(p, q)$ is linearly stable at least for some $S$ and $T$.

Let us consider the linearized equation of $(2.2)$ around $(\varphi(x), \psi(x))$ :

$$
\left\{\begin{array}{l}
C U_{x x}+f_{u} U+f_{v} V=0, \\
D V_{x x}+g_{u} U+g_{v}=0,
\end{array} \quad x \in \mathbf{R},\right.
$$

where $f_{u}, f_{v}, g_{u}, g_{v}$ are Jacobian matrices evaluated at $(u, v)=(\varphi(x), \psi(x))$. Differentiating (2.3) by $x$, we see that $(U, V)=\left(\varphi_{x}, \psi_{x}\right)$ is a bounded solution of $(2.4)$. Here we impose the following nondegeneracy condition on the standing pulse solution:

(ND) $(U, V)=\left(\varphi_{x}, \psi_{x}\right)$ is the unique bounded solution up to multiplication by constants. 
By (1.2), we have

$$
\begin{gathered}
f_{u}:=\nabla_{u} f=\left(\frac{\partial f_{i}}{\partial u_{j}}\right)=\left(+\frac{\partial^{2} H}{\partial u_{i} \partial u_{j}}\right), \\
g_{v}:=\nabla_{v} g=\left(\frac{\partial g_{i}}{\partial v_{j}}\right)=\left(-\frac{\partial^{2} H}{\partial v_{i} \partial v_{j}}\right), \\
f_{v}:=\nabla_{v} f=\left(\frac{\partial f_{i}}{\partial v_{j}}\right)=\left(+\frac{\partial^{2} H}{\partial u_{i} \partial v_{j}}\right), \\
g_{u}:=\nabla_{u} g=\left(\frac{\partial g_{i}}{\partial u_{j}}\right)=\left(-\frac{\partial^{2} H}{\partial u_{j} \partial v_{i}}\right),
\end{gathered}
$$

so that

$$
f_{u}={ }^{t} f_{u}, \quad f_{v}=-{ }^{t} g_{u}, \quad g_{v}={ }^{t} g_{v} .
$$

Hence the adjoint system of (2.4) is written as

$$
\left\{\begin{array}{l}
C U_{x x}+f_{u} U-f_{v} V=0, \\
D V_{x x}-g_{u} U+g_{v} V=0,
\end{array} \quad x \in \mathbf{R} .\right.
$$

Therefore, $(U, V)=\left(\varphi_{x},-\psi_{x}\right)$ is a bounded solution of (2.5), and it is unique up to multiplication by constants if (ND) holds.

Here we define orientations of standing pulse solutions as follows. We rewrite $(2.2)$ to a $(2 m+2 n)$-dimensional dynamical system

$$
\frac{d}{d x} w=h(w)
$$

where

$$
w=\left(\begin{array}{l}
u \\
v \\
u_{x} \\
v_{x}
\end{array}\right), \quad h(w)=\left(\begin{array}{c}
u_{x} \\
v_{x} \\
-C^{-1} f(u, v) \\
-D^{-1} g(u, v)
\end{array}\right)
$$

Clearly

$$
\bar{p}:=\left(\begin{array}{c}
p \\
q \\
0 \\
0
\end{array}\right) \in \mathbf{R}^{2 m+2 n}
$$

is an equilibrium point of (2.6). It follows from (2.3) that

$$
w=\Phi(x):=\left(\begin{array}{c}
\varphi(x) \\
\psi(x) \\
\varphi_{x}(x) \\
\psi_{x}(x)
\end{array}\right)
$$


is a solution of (2.6) which connects $\bar{p}$ with itself. Such a solution is called a homoclinic solution, and its trajectory in the $(2 m+2 n)$-dimensional phase space is called a homoclinic orbit.

Define a function $J: \mathbf{R}^{2 m+2 n} \rightarrow \mathbf{R}$ by

$$
J(w):=\frac{1}{2} C u_{x} \cdot u_{x}-\frac{1}{2} D v_{x} \cdot v_{x}+H(u, v),
$$

where "." denotes the usual inner product of two vectors. It is easy to show that if $w$ satisfies $(2.6)$, then

$$
J(w(x)) \equiv \text { Const. for all } x .
$$

Let $M$ be a $(2 m+2 n-1)$-dimensional hypersurface in $\mathbf{R}^{2 m+2 n}$ given by

$$
M=\left\{w \in \mathbf{R}^{2 m+2 n} ; J(w)=H(p, q)\right\},
$$

and let $M^{s}, M^{u}$ be a stable manifold and an unstable manifold, respectively, with respect to the equilibrium $\bar{p}$. It is clear that the equilibrium point $\bar{p}$ is on these manifolds. It is also clear that there exists a homoclinic solution connecting $\bar{p}$ with itself if and only if

$$
M^{u} \cap M^{s} \neq \emptyset
$$

and the homoclinic orbit is entirely contained in the intersection manifold. Since any solution on $M^{u}$ converges to $\bar{p}$ as $x \rightarrow-\infty$, we have

$$
J(w) \equiv H(p, q) \quad \text { for } w \in M^{u} .
$$

Similarly we have

$$
J(w) \equiv H(p, q) \quad \text { for } w \in M^{s} .
$$

Therefore, $M^{u}$ and $M^{s}$ are submanifolds of $M$.

Let us study properties of the stable and unstable manifolds. If $w(x)$ is in a small neighborhood of $\bar{p}$, then $W(x)=w(x)-\bar{p}$ satisfies approximately the linearized equation

$$
\frac{d}{d x} W=h_{w}(\bar{p}) W
$$

where $h_{w}$ is a Jacobian matrix of $h$ given by

$$
h_{w}(w)=\left(\begin{array}{cccc}
0 & 0 & I_{m} & 0 \\
0 & 0 & 0 & I_{n} \\
-C^{-1} f_{u}(u, v) & 0 & 0 & 0 \\
0 & -D^{-1} g_{v}(u, v) & 0 & 0
\end{array}\right) .
$$

Let $\alpha_{i}, i=1,2, \ldots, 2 m+2 n$, be characteristic roots of $h_{w}(\bar{p})$. Since $h_{w}$ is of the form as above, we may assume

$$
0 \leq \Re\left\{\alpha_{1}\right\} \leq \Re\left\{\alpha_{2}\right\} \leq \cdots \leq \Re\left\{\alpha_{m+n}\right\}
$$


and

$$
\alpha_{m+n+i}=-\alpha_{i}, \quad i=1,2, \ldots, m+n .
$$

It can be shown that if $(u, v)=(p, q)$ is a stable steady state of $(2.1)$, then $\Re\left\{\alpha_{i}\right\} \neq 0$ for all $i$ (see [9, Lemma 3.1]).

Let $a_{1}, a_{2}, \ldots, a_{m+n} \in \mathbf{R}^{2 m+n}$ be independent vectors which belong to the eigenspace of $h_{w}(\bar{p})$ associated with $\alpha_{1}, \ldots, \alpha_{m+n}$, and let $a_{m+n+1}, \ldots, a_{2 m+2 n} \in$ $\mathbf{R}^{2 m+2 n}$ be independent vectors which belong to the eigenspace of $h_{w}(\bar{p})$ associated with $\alpha_{m+n+1}, \ldots, \alpha_{2 m+2 n}$. Then $M^{u}$ is an $(m+n)$-dimensional manifold which is tangent to $a_{1}, a_{2}, \ldots, a_{m+n}$ at $\bar{p}$, and $M^{s}$ is an $(m+n)$-dimensional manifold which is tangent to $a_{m+n+1}, \ldots, a_{2 m+2 n}$ at $\bar{p}$. We take these vectors as bases of $M^{u}$ and $M^{s}$, respectively. Now the orientations of $M^{u}$ and $M^{s}$ can be defined by these bases. We assume without losing generality that

$$
\operatorname{det}\left(a_{1}, \ldots, a_{n}, a_{m+n+1}, \ldots, a_{2 m+2 n}\right)=1,
$$

which introduces a relationship between the orientations of $M^{u}$ and $M^{s}$.

Let us consider the linearized equation of (2.6) around $w=\Phi(x)$

$$
\frac{d}{d x} W=h_{w}(\Phi(x)) W
$$

and its adjoint equation

$$
\frac{d}{d x} W=-{ }^{t} h_{w}(\psi(x)) W
$$

Differentiating (2.6) by $x$, we see that $W=\Phi_{x}(x)$ satisfies (2.8). Then it follows from (2.5) and (2.7) that

$$
W^{*}(x):=\left(\begin{array}{c}
+C \varphi_{x x} \\
-D \psi_{x x} \\
-C \varphi_{x} \\
+D \psi_{x}
\end{array}\right)
$$

satisfies (2.9). Since

$$
-\nabla_{w} J(\Phi)=-\left(\begin{array}{c}
+f(\varphi, \psi) \\
-g(\varphi, \psi) \\
+C \varphi_{x} \\
-D \psi_{x}
\end{array}\right)=\left(\begin{array}{c}
+C \varphi_{x x} \\
-D \psi_{x x} \\
-C \varphi_{x} \\
+D \psi_{x}
\end{array}\right)=W^{*}
$$

$W^{*}(x)$ must be a normal vector of $M$ (and hence a normal vector of $M^{u}$ and $M^{s}$ ) at $w=\Phi(x)$ which points the direction where $J(w)$ becomes smaller. Thus, the nondegeneracy condition (ND) implies that the intersection of normal spaces of $M^{u}$ and $M^{v}$ at $\Phi(x)$ is one-dimensional for any $x$. In other words, $M^{u}$ and $M^{v}$ intersects transversally in $M$.

Let $\left\{s_{1}, \ldots, s_{m+n}\right\}$ be a positively oriented basis of the tangent space of $M^{u}$ at $v=\psi(0)$, and let $\left\{s_{m+n+1}, \ldots, s_{2 m+2 n}\right\}$ be a positively oriented basis of the tangent 
space of $M^{s}$ at $v=\psi(0)$. Without loss of generality, we may assume $s_{1}=s_{2 m+2 n}=$ $\Phi_{x}(0)$. By (ND), the vectors $s_{1}, \ldots, s_{2 m+2 n-1}$ are linearly independent.

Let $W_{i}(x), i=1,2, \ldots, 2 m+2 n$, be solutions of (2.8) subject to the initial conditions

$$
W_{i}(0)=s_{i}
$$

Then the solutions satisfy

$$
\begin{aligned}
& W_{i}(x) \rightarrow 0 \quad \text { as } x \rightarrow-\infty, \quad i=1,2, \ldots, m+n, \\
& W_{i}(x) \rightarrow 0 \quad \text { as } x \rightarrow+\infty, \quad i=m+n+1, \ldots, 2 m+2 n .
\end{aligned}
$$

In particular $W_{1}(x)$ and $W_{2 m+2 n}(x)$ satisfy

$$
W_{1}(x) \equiv \Phi_{x}(x) \equiv W_{2 m+2 n}(x) .
$$

We note that the set $\left\{W_{1}(x), \ldots, W_{2 m+2 n-1}(x)\right\}$ is a basis of the tangent space of $M$ at $v=\psi(x)$, the set $\left\{W_{1}(x), \ldots, W_{m+n}(x)\right\}$ is a positively oriented basis of the tangent space of $M^{u}$ at $w=\Psi(x)$, and the set $\left\{W_{m+n+1}(x), \ldots, W_{2 m+2 n}(x)\right\}$ is a positively oriented basis of the tangent space of $M^{s}$ at $w=\Phi(x)$.

Let $W_{0}(x)$ be a solution of (2.8) subject to the initial condition

$$
W_{0}(0)=W^{*}(0) \text {. }
$$

Since $W^{*}(0)$ is a normal vector of both $M^{u}$ and $M^{v}, W_{0}(x)$ must satisfy

$$
\left|W_{0}(x)\right| \rightarrow \infty \quad \text { as } x \rightarrow \pm \infty .
$$

We note that $W_{0}(x)$ is transversal to the tangent space of $M$ at $w=\Phi(x)$. follows.

Now we give a definition of an orientation of the homoclinic solution $\Phi(x)$ as

Definition The solution $(u, v)=(\varphi(x), \psi(x))$ is said to be positively oriented (resp. negatively oriented) if

$$
\operatorname{det}\left(s_{1}, \ldots, s_{n}, s_{m+n+1}, \ldots, s_{2 m+2 n-1}, W^{*}(0)\right)
$$

is positive (resp. negative).

Now we are in a position to state our main result.

THEOREM 2.1. Let $(u, v)=(\varphi(x), \psi(x))$ be a standing pulse solution of the skew-gradient system (2.1). If $(\varphi(x), \psi(x))$ is positively (resp. negatively) oriented, then it is unstable for any $S$ and $T$ with

$$
\begin{gathered}
\int_{\mathbf{R}} S \varphi_{x} \cdot \varphi_{x} d x<\int_{\mathbf{R}} T \psi_{x} \cdot \psi_{x} d x \\
\left(\text { resp. } \int_{\mathbf{R}} S \varphi_{x} \cdot \varphi_{x} d x>\int_{\mathbf{R}} T \psi_{x} \cdot \psi_{x} d x\right) .
\end{gathered}
$$

This theorem is proved in [9] by analyzing the eigenvalue problem

$$
\left\{\begin{array}{l}
\lambda S U=C U_{x x}+f_{u} U+f_{v} V, \\
\lambda T V=D V_{x x}+g_{u} U+g_{v} V .
\end{array} \quad x \in \mathbf{R}\right.
$$


The complex number $\lambda$ is called an eigenvalue if this system has a uniformly bounded solution, and the standing pulse solution is unstable if there exists an eigenvalue with a positive real part. To determine the location of eigenvalues, the so-called Evans function [2] is used in [9]. We omit details here and refer the reader to [9].

3. Eckhaus Instability. In this section, based on [5], we consider the skewgradient system on $\mathbf{R}$ written as

$$
\left\{\begin{array}{l}
S u_{t}=C u_{x x}+f(u, v), \\
T v_{t}=D v_{x x}+g(u, v),
\end{array} \quad x \in \mathbf{R} .\right.
$$

Let $(u, v)=(\varphi(x ; s), \psi(x ; s))$ be a family of spatially periodic stationary solutions of (3.1) parametrized by $s$ with its minimal spatial period $l(s)$, that is, $(\varphi(x ; s), \psi(x ; s))$ satisfies

$$
\left\{\begin{array}{l}
C \varphi_{x x}(x ; s)+f(\varphi(x ; s), \psi(x ; s))=0, \\
D \psi_{x x}(x ; s)+g(\varphi(x ; s), \psi(x ; s))=0, \quad x \in \mathbf{R} . \\
\varphi(x ; s)=\varphi(x+l(s) ; s) .
\end{array}\right.
$$

The aim of this section is to investigate the stability of $(\varphi(x ; s), \psi(x ; s))$ in the space of uniformly bounded functions on $\mathbf{R}$. Namely, we consider the linearized eigenvalue problem

$$
\left\{\begin{array}{l}
\lambda S U=C U_{x x}+f_{u} U+f_{v} V, \\
\lambda T V=D V_{x x}+g_{u} U+g_{v} V,
\end{array} \quad x \in \mathbf{R},\right.
$$

where $f_{u}, f_{v}, g_{u}$ and $g_{v}$ are evaluated at $(u, v)=(\varphi(x ; s), \psi(x ; s))$. We denote the spectrum of (3.3) by $\Lambda(s)$. Differentiating (3.2) with respect to $x$, we immediately find that $\lambda=0$ is an eigenvalue of $(3.3)$ with an eigenfunction $(U, V)=\left(\varphi_{x}(x ; s), \psi_{x}(x ; s)\right)$. As is well-known, the spectrum near zero often determines the stability/instability of stationary solutions in dissipative systems.

Setting

$$
W=\left(\begin{array}{c}
U \\
V \\
U_{x} \\
V_{x}
\end{array}\right)
$$

we can rewrite (3.3) as a system of first order system

$$
\frac{d}{d x} W=(B(x ; s)+\lambda K) W
$$

where $B(x ; s)$ and $K$ are $(2 m+2 n) \times(2 m+2 n)$-matrices given by

$$
B(x ; s)=\left(\begin{array}{cccc}
0 & 0 & I_{m} & 0 \\
0 & 0 & 0 & I_{n} \\
-C^{-1} f_{u} & 0 & 0 & 0 \\
0 & -D^{-1} g_{v} & 0 & 0
\end{array}\right)
$$


and

$$
K=\left(\begin{array}{cccc}
0 & 0 & 0 & 0 \\
0 & 0 & 0 & 0 \\
C^{-1} S & 0 & 0 & 0 \\
0 & D^{-1} T & 0 & 0
\end{array}\right)
$$

respectively. Clearly, $B(x ; s)$ is an $l(s)$-periodic function of $x$. We will consider conditions so that (3.4) has a uniformly bounded solution for some $\Re\{\lambda\}>0$. To do so, it suffices to consider the monodromy matrix $\Phi(l(s) ; \lambda, s): \mathbf{C}^{2 m+2 n} \rightarrow \mathbf{C}^{2 m+2 n}$ of (3.4), where $\Phi(x ; \lambda, s)$ is the fundamental matrix of $(3.4)$ defined by

$$
\frac{d}{d x} \Phi(x ; \lambda, s)=(B(x ; s)+\lambda K) \Phi(x ; \lambda, s), \quad \Phi(0 ; \lambda, s)=I_{2 m+2 n} .
$$

Then, $\lambda$ becomes an eigenvalue of (3.4) if and only if $\Phi(l(s) ; \lambda, s)$ has an eigenvalue whose absolute value is equal to one.

Differentiating (3.2) with respect to $x$ and $s$, we immediately find that $\Phi(l(s) ; 0, s)$ has a degenerate eigenvalue 1 . Moreover, we can show that if $\mu$ is an eigenvalue of $\Phi(l(s) ; \lambda, s)$, then $1 / \mu$ is also an eigenvalue of $\Phi(l(s) ; \lambda, s)$. Noting these facts, we consider conditions such that the degenerate eigenvalue 1 of $\Phi(l(s) ; \lambda, s)$ splits into two simple eigenvalues with the absolute value equal to one when $\lambda$ moves from the origin into the right-half plane. In this case, the stationary solution is unstable with some spatially modulating unstable mode, and such instability is called the Eckhaus instability.

Here we introduce a function that will play an important role in our stability analysis. From (3.2), we see that

$$
J[\varphi, \psi]:=\frac{1}{2} C \varphi_{x} \cdot \varphi_{x}-\frac{1}{2} D \psi_{x} \cdot \psi_{x}+H(\varphi, \psi)
$$

is constant in $x$. In other words, $J[\varphi, \psi] \equiv$ Const. is a first integral of the equation in (3.2). Thus we can define a function $J(s)$ by

$$
J(s):=J[\varphi(\cdot ; s), \psi(\cdot ; s)]
$$

on the one-parameter family of stationary solutions $(\varphi(x ; s), \psi(x ; s)$ of $(3.1)$. We note that $d J(s) / d s$ is computed as

$$
\begin{array}{rl}
\frac{d}{d s} J(s)= & C \varphi_{x}(x ; s) \cdot \varphi_{x s}(x ; s)-D \psi_{x}(x ; s) \cdot \psi_{x s}(x ; s) \\
& -\left\{C \varphi_{x x}(x ; s) \cdot \varphi_{s}(x ; s)-D \psi_{x x}(x ; s) \cdot \psi_{s}(x ; s)\right\} \\
=C & C \varphi_{x}(0 ; s) \cdot \varphi_{x s}(0 ; s)-D \psi_{x}(0 ; s) \cdot \psi_{x s}(0 ; s) \\
& -\left\{C \varphi_{x x}(0 ; s) \cdot \varphi_{s}(0 ; s)-D \psi_{x x}(0 ; s) \cdot \psi_{s}(0 ; s)\right\}
\end{array}
$$

Now, our main result is roughly stated as follows. (See [5] for a precise statement of the result, ).

THEOREM 3.1. The Eckhaus instability occurs if and only if

$$
d J / d l:=\frac{d J(s)}{d s} / \frac{d l(s)}{d s}
$$


and

$$
I(s):=\int_{0}^{l(s)}\left\{S \varphi_{x}(x ; s) \cdot \varphi_{x}(x ; s)-T \psi_{x}(x ; s) \cdot \psi_{x}(x ; s)\right\} d x
$$

have the same sign.

It should be noted that $(\varphi(x ; s), \psi(x ; s))$ is not required to be small in this theorem. This theorem implies that a stability-instability transition must occur at extremal points of $J(s)$ when $l(s)$ is strictly monotone in $s$. Thus, a first integral $J(s)$ is an index of the instability property of periodic stationary solutions.

Our proof of Theorem 3.1 is based on a careful analysis of the linearized eigenvalue problem (3.3) when $\lambda$ varies around $\lambda=0$. One of the advantages to introduce the skew-gradient structure is that the analysis of an adjoint system for (3.3) becomes easier than that for systems without gradient/skew-gradient structure. In fact, we can express solutions of the adjoint system for (3.4) by using solutions of (3.4). This advantage helps us to derive a rather explicit description for the behavior of eigenvalues of the monodromy matrix $\Phi(l(s) ; \lambda, s)$.

Here we give a few applications of Theorem 3.1 to demonstrate its usefulness.

Application. Let us consider the following reaction-diffusion system of activator-inhibitor type

$$
\left\{\begin{array}{l}
\tau_{1} u_{t}=d_{1} u_{x x}+\alpha u-u^{3}-v \\
\tau_{2} v_{t}=d_{2} v_{x x}+u-\gamma v
\end{array}\right.
$$

where $\tau_{1}, \tau_{2}, d_{1}, d_{2}, \alpha, \gamma>0$. By setting

$$
H(u, v)=\frac{1}{2} \alpha u^{2}-\frac{1}{4} u^{4}-u v+\frac{1}{2} \gamma v^{2},
$$

(3.5) is written as

$$
\left\{\begin{array}{c}
\tau_{1} u_{t}=d_{1} u_{x x}+\frac{\partial H}{\partial u} \\
\tau_{2} v_{t}=d_{2} v_{x x}-\frac{\partial H}{\partial v}
\end{array}\right.
$$

so that (3.5) has skew-gradient structure. Applying a standard argument based on the Liapunov-Schmidt method, we can construct a family of spatially periodic stationary solutions of (3.5) when $0<\alpha<\gamma$ and $\alpha \gamma<1$ as follows. Let us define

$$
\hat{d}_{2}\left(s^{2}\right)=\frac{1}{s^{2}\left(\alpha-d_{1} s^{2}\right)}-\frac{\gamma}{s^{2}}
$$

Assume $0<s^{2}<\alpha / d_{1}$ and $\hat{d}_{2}\left(s^{2}\right)<d_{2}$. Then, for $\left(s^{2}, d_{2}\right)$ near $\left(s^{2}, \hat{d}_{2}\left(s^{2}\right)\right)$, we have

$$
\left(\begin{array}{c}
\varphi(x ; s) \\
\psi(x ; s)
\end{array}\right)=2 \sqrt{\frac{\left(d_{2}-\hat{d}_{2}\left(s^{2}\right) s^{2}\right.}{3}} \cos (s x)\left(\begin{array}{c}
c(s) \\
c(s)^{2}
\end{array}\right)+\text { h.o.t. }
$$

where $c(s):=\alpha-d_{1} s^{2}$. Neglecting higher order terms and noting $l(s)=2 \pi / s$, direct calculation yields

$$
\begin{aligned}
I(s) & =\int_{0}^{l(s)}\left\{\tau_{1} \varphi_{x}(x ; s)^{2}-\tau_{2} \psi_{x}(x ; s)^{2}\right\} d x \\
& =\frac{4 \pi s^{3}}{3}\left(d_{2}-\hat{d}_{2}\right) c(s)^{2}\left(\tau_{1}-\tau_{2} c(s)^{2}\right) .
\end{aligned}
$$


On the other hand, the computation of $d J / d s$ is extremely complicated, but the result turns out to be

$$
\frac{d}{d s} J(s)=-\frac{4}{3} s^{3} K\left(s^{2}, d_{2}\right)
$$

where $K\left(s^{2}, d_{2}\right)$ is a polynomial of degree four in $s^{2}$ and quadratic degree in $d_{2}$. Thus we obtain

$$
d J / d l=\frac{2 s^{5}}{3 \pi} K\left(s^{2}, d_{2}\right)
$$

Consequently, we have the following result.

THEOREM 3.2. Let $(u, v)=(\varphi(x ; s), \psi(x ; s)$ be a stationary solution of $(3.5)$ of the form (3.6). Suppose that $K\left(s^{2}, d_{2}\right)>0$ (resp. $K\left(s^{2}, d_{2}\right)<0$ ). If $\tau_{1}>c(s)^{2} \tau_{2}$ (resp. $\left.\tau_{1}<\tau_{2} c(s)^{2}\right)$, then $(u, v)=\varphi(x ; s)$ is unstable.

Although this results does not necessarily guarantee the stability of bifurcating stationary solutions in the region $\left\{\left(s^{2}, d_{2}\right) \mid K\left(s^{2}, d_{2}\right)<0\right\}$, numerical simulations suggest that these stationary solutions are stable when $\tau_{1}>c(s)^{2} \tau_{2}$. Since the sign of $K\left(s^{2}, d_{2}\right)$ is independent of $\tau_{1}$ and $\tau_{2}$, this result implies the following by the aid of numerical simulations: the bifurcating stationary solutions are stable in the region $\left\{\left(s^{2}, d_{2}\right) \mid K\left(s^{2}, d_{2}\right)<0\right\}$ when the ratio of time constant coefficients of activator and inhibitor $\tau_{1} / \tau_{2}$ is large, whereas these stationary solutions become unstable and various complicated behavior of solutions can be observed when $\tau_{1} / \tau_{2}$ is small. For example, when $d_{1}$ is small, the stationary solutions lose their stability, and there appear metastable patterns. On the other hand, when $d_{1}$ is large, there appear oscillatory patterns which cannot be observed in gradient systems.

Next we briefly mention about the Gierer-Meinhardt system (1.4), where the parameters are assumed to satisfy $p>1, q, r>0, s, \sigma \geq 0$ and

$$
\frac{p-1}{q}<\frac{r}{s+1}
$$

Then there exists a unique positive spatially homogeneous stationary solution. As is noted in the introduction, the Gierer-Meinhardt system has skew-gradient structure when $p+1=r$ and $q+1=s$. Recalling [8], in a manner similar to (3.5), we can construct bifurcating stationary solutions with spatially periodic structure around the unique positive spatially homogeneous stationary solution. In a manner similar to the above, we can also obtain a criterion for the Eckhaus instability as in Theorem 3.2.

4. Mini-Maximizers. In this section, based on [10], we study the skew-gradient system

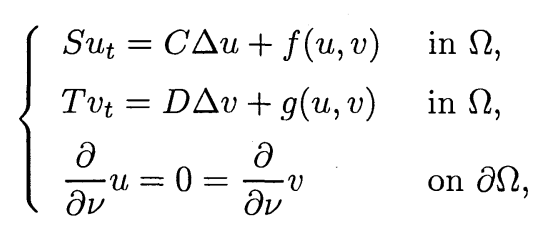

where $\Omega$ is a bounded domain in $\mathbf{R}^{N}$ with smooth boundary $\partial \Omega, \partial / \partial \nu$ stands for the outward normal derivative on $\partial \Omega$. Any steady state $(u, v)=(\varphi(x), \psi(x))$ of $(4.1)$ 
satisfies the system of elliptic equations

$$
\begin{cases}C \Delta \varphi+f(\varphi, \psi)=0, & \text { in } \Omega, \\ D \Delta \psi+g(\varphi, \psi)=0 & \text { in } \Omega, \\ \frac{\partial}{\partial \nu} \varphi=0=\frac{\partial}{\partial \nu} \psi & \text { on } \partial \Omega .\end{cases}
$$

We note that the solution of this problem corresponds to a critical point of the functional

$$
E[u, v]:=\int_{\Omega}\left\{\frac{1}{2}\langle C \nabla u, \nabla u\rangle-\frac{1}{2}\langle D \nabla v, \nabla v\rangle-H(u, v)\right\} d x,
$$

where $\nabla$ is a gradient operator with respect to $x$ and

$$
\langle C \nabla u, \nabla u\rangle:=\sum_{i=1}^{m} c_{i}\left|\nabla u_{i}\right|^{2}, \quad\langle D \nabla v, \nabla v\rangle:=\sum_{i=1}^{n} d_{i}\left|\nabla v_{i}\right|^{2},
$$

with $C=\operatorname{diag}\left(c_{1}, c_{2}, \ldots, c_{m}\right)$ and $D=\operatorname{diag}\left(d_{1}, d_{2}, \ldots, d_{m}\right)$. In fact, (4.2) is the Euler-Lagrange equation for $E[u, v]$. We say that $(u, v)=(\varphi, \psi)$ is a mini-maximizer of $E[u, v]$ if $u=\varphi$ is a minimizer of $E[u, \psi]$ and $v=\psi$ is a maximizer of $E[\varphi, v]$, that is,

$$
E[U, \psi] \geq E[\varphi, \psi]
$$

for any $U$ in a neighborhood of $\varphi$ in $H^{1}(\Omega)$, and

$$
E[\varphi, V] \leq E[\varphi, \psi]
$$

for any $V$ in a neighborhood of $\psi$ in $H^{1}(\Omega)$. The purpose of this section is to study the relation between a stability property of $(u, v)=(\varphi, \psi)$ as a steady state of (4.1) and a mini-maximizing property as a critical point of $E[u, v]$.

When $v$ is fixed to $\psi(x)$ in the first equation of (4.1), then we have a system for $u$

$$
\begin{cases}S u_{t}=C \Delta u+f(u, \psi) & \text { in } \Omega \\ \frac{\partial}{\partial \nu} u=0 & \text { on } \partial \Omega\end{cases}
$$

For any solution $u(x, t)$ of this equation, we have

$$
\begin{aligned}
\frac{d}{d t} E[u(x, t), \psi(x)] & =\int_{\Omega}\left\{\left\langle C \nabla u, \nabla u_{t}\right\rangle-f(u, \psi) \cdot u_{t}\right\} d x \\
& =\int_{\Omega}\left\{-C \Delta u \cdot u_{t}-f(u, \psi) \cdot u_{t}\right\} d x \\
& =-\int_{\Omega} S u_{t} \cdot u_{t} d x \leq 0 .
\end{aligned}
$$

Hence (4.3) describes a gradient flow of $E[u, \psi]$. Therefore, $u=\varphi$ is a steady state of (4.3) if and only if $u=\varphi$ is a critical point of $E[u, \psi]$, and is stable if and only if it is a local minimizer of $E[u, \psi(x)]$. 
Similarly, when $u$ is fixed to $\varphi(x)$ in the second equation of (4.1), then we have a system for $v$

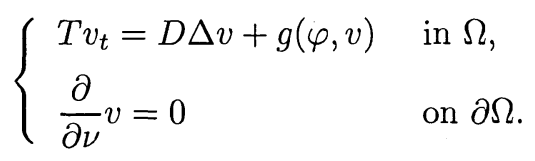

For any solution $v(x, t)$ of this equation, we have

$$
\begin{aligned}
\frac{d}{d t} E[\varphi(x), v(x, t)] & =\int_{\Omega}\left\{-\left\langle D \nabla v, \nabla v_{t}\right\rangle+g(\varphi, v) \cdot v_{t}\right\} d x \\
& =\int_{\Omega}\left\{D \Delta v \cdot v_{t}+g(\varphi, v) \cdot v_{t}\right\} d x \\
& =\int_{\Omega} T v_{t} \cdot v_{t} d x \geq 0 .
\end{aligned}
$$

Hence (4.4) describes a gradient flow of $-E[\varphi, v]$. Therefore, $v=\psi$ is a steady state of (4.4) if and only if $v=\psi$ is a critical point of $E[\varphi, v]$, and is stable as a steady state of (4.4) if and only if it is a maximizer of (4.4).

Even if $u=\varphi$ is a minimizer of $E[u, \psi]$ and $v=\psi$ is a maximizer of $E[\varphi, v]$, due to the interaction between $u$ and $v$, it does not automatically mean that $(u, v)=(\varphi, \psi)$ is stable as a steady state of (4.1). In fact, if $(u, v)$ is a solution of $(4.1)$, then

$$
\begin{aligned}
\frac{d}{d t} & E[u(x, t), v(x, t)] \\
& =\int_{\Omega}\left\{\frac{1}{2}\left\langle C \nabla u, \nabla u_{t}\right\rangle-\frac{1}{2}\left\langle D \nabla v, \nabla v_{t}\right\rangle-f(u, v) \cdot u_{t}+g(u, v) \cdot v_{t}\right\} d x \\
& =\int_{\Omega}\left\{-S u_{t} \cdot u_{t}+T v_{t} \cdot v_{t}\right\} d x .
\end{aligned}
$$

Hence $E[u, v]$ is not necessarily nonincreasing or nondecreasing in $t$, and cannot be used as a Liapunov functional. Thus, roughly speaking, the reaction-diffusion system with skew-gradient structure is a sort of activator-inhibitor system which consists of two gradient systems coupled in a skew-symmetric way.

Let $(\varphi, \psi)$ be a solution of (4.2). As is well-known [4], stability of $(u, v)=(\varphi, \psi)$ as a steady state of (4.1) can be determined by the eigenvalue problem

$$
\left\{\begin{array}{l}
\lambda S U=C \Delta U+f_{u} U+f_{v} V, \\
\lambda T V=D \Delta V+g_{u} U+g_{v} V,
\end{array}\right.
$$

on $\Omega$ under the Neumann boundary conditions, where $f_{u}, f_{v}, g_{u}$ and $g_{v}$ are evaluated at $(\varphi, \psi)$. Since this is not a self-adjoint eigenvalue problem, there may exist complex eigenvalues. Usually, in such a situation, it is extremely difficult to locate the eigenvalues. However, if $(u, v)=(\varphi, \psi)$ is a mini-maximizer of $E[u, v]$, we can show by using the skew-gradient structure that any eigenvalue has a negative real part regardless of the choice of $S$ and $T$. Conversely, if $(u, v)=(\varphi, \psi)$ is not a mini-maximizer of $E[u, v]$, then there exists a positive eigenvalue for some $S$ and $T$.

A critical point $u=\varphi$ of $E[u, \psi]$ is said to be nondegenerate if the linearized operator

$$
\mathcal{A}:=C \Delta+f_{u}
$$


is invertible. Similarly, a critical point $v=\psi$ of $E[\varphi, v]$ is said to be nondegenerate if the linearized operator

$$
\mathcal{B}:=D \Delta+g_{v}
$$

is invertible. Finally, we say that $(u, v)=(\varphi, \psi)$ is a nondegenerate critical point of $E[u, v]$ if $u=\varphi$ and $v=\psi$ are nondegenerate critical points of $E[u, \psi]$ and $E[\varphi, v]$, respectively.

Next, we describe some properties of the eigenvalue problem

$$
\begin{cases}\lambda S U=\mathcal{A} U & \text { in } \Omega, \\ \frac{\partial}{\partial \nu} U=0 & \text { on } \partial \Omega .\end{cases}
$$

Since $f_{u}={ }^{t} f_{u}$, the operator $\mathcal{A}$ is self-adjoint. Hence, by the variational principle, we have the following result.

LEMMA 4.1. All eigenvalues of (4.8) are real. Moreover, there exists a maximal eigenvalue $\lambda^{u}$ with finite multiplicity that is characterized by

$$
\lambda^{u}=\sup _{U \in H^{1}(\Omega)} \frac{\int_{\Omega}\left\{-\langle C \nabla U, \nabla U\rangle+f_{u} U \cdot U\right\} d x}{\int_{\Omega} S U \cdot U d x},
$$

and the supremum is attained by an eigenfunction of (4.8) associated with $\lambda^{u}$.

We see from this lemma that the maximal eigenvalue $\lambda^{u}$ depends on $S$ but its sign does not depend on $S$. We say that $u=\varphi$ is linearly stable if $\lambda^{u}<0$ and linearly unstable if $\lambda^{u}>0$ as a steady state of (4.3).

Next, we consider the eigenvalue problem

$$
\begin{cases}\lambda T V=\mathcal{B} V & \text { in } \Omega, \\ \frac{\partial}{\partial \nu} V=0 & \text { on } \partial \Omega .\end{cases}
$$

The following lemmas can be obtained in the same manner as Lemma 4.1.

Lemma 4.2. All eigenvalues of (4.9) are real. Moreover, there exists a maximal eigenvalue $\lambda^{v}$ with finite multiplicity that is characterized by

$$
\lambda^{v}=\sup _{V \in H^{1}(\Omega)} \frac{\int_{\Omega}\left\{-\langle D \nabla V, \nabla V\rangle+g_{v} V \cdot V\right\} d x}{\int_{\Omega} T V \cdot V d x},
$$

and the supremum is attained by an eigenfunction of (4.8) associated with $\lambda^{v}$.

We note that the maximal eigenvalue $\lambda^{v}$ depends on $T$ but its sign does not depend on $T$. We say that $u=\psi$ is linearly stable if $\lambda^{v}<0$ and is linearly unstable if $\lambda^{v}>0$ as a steady state of (4.4). We note that $(\varphi, \psi)$ is a nondegenerate mini-maximizer of $E[u, v]$ if and only if both $u=\varphi$ and $v=\psi$ are linearly stable. 
Let $(\varphi, \psi)$ be a solution of (4.2). In order to study the stability of $(u, v)=(\varphi, \psi)$ as a steady state of (4.1), we rewrite the eigenvalue problem (4.5) as

$$
\left\{\begin{array}{l}
\lambda S U=\mathcal{A} U+f_{v} V \\
\lambda T V=\mathcal{B} V+g_{u} U
\end{array}\right.
$$

where $\mathcal{A}$ and $\mathcal{B}$ are the operators defined by (4.6) and (4.7), respectively. We note that the eigenvalue $\lambda$ and the eigenfunction $(U, V)$ of (4.10) may be complex-valued. We say that $(u, v)=(\varphi, \psi)$ is linearly stable as a steady state of $(4.1)$ if for some $\delta>0$, all eigenvalues of (4.10) satisfy $\Re\{\lambda\}<-\delta$. Conversely, the steady state is said to be linearly unstable if there exists an eigenvalue of (4.10) with a positive real part. It is well-known [4] that the linearly stable (resp. unstable) steady state is stable (resp. unstable) in the sense of Lyapunov.

First we consider the case where $(\varphi, \psi)$ is a nondegenerate mini-maximizer of $E[u, v]$.

THEOREM 4.1. Let $(u, v)=(\varphi, \psi)$ be a nondegenerate mini-maximizer of $E[u, v]$. Then, for any $S$ and $T,(u, v)=(\varphi, \psi)$ is linearly stable as a steady state of (4.1).

The proof is given as follows. From

$$
\left\{\begin{array}{l}
\lambda S U=\mathcal{A} U+f_{v} V \\
\bar{\lambda} T \bar{V}=\mathcal{B} \bar{V}+g_{u} \bar{U}
\end{array}\right.
$$

and $f_{v}=-{ }^{t} g_{u}$, we have

$$
\lambda \int_{\Omega} S U \cdot \bar{U} d x+\bar{\lambda} \int_{\Omega} T \bar{V} \cdot V d x=\int_{\Omega} \mathcal{A} U \cdot \bar{U} d x+\int_{\Omega} \mathcal{B} \bar{V} \cdot V d x
$$

Here, the integrals

$$
\int_{\Omega} S U \cdot \bar{U} d x, \quad \int_{\Omega} T \bar{V} \cdot V d x
$$

are positive. On the other hand, by partial integration, we have

$$
\int_{\Omega} \mathcal{A} U \cdot \bar{U} d x=\int_{\partial \Omega} C \frac{\partial}{\partial \nu} U \cdot \bar{U} d x+\int_{\Omega}\left\{-\langle C \nabla U, \nabla \bar{U}\rangle+f_{u} U \cdot \bar{U}\right\} d x .
$$

The first term in the right-hand side vanishes due to the Neumann boundary condition, and the second term satisfies

$$
\int_{\Omega}\left\{-\langle C \nabla U, \nabla \bar{U}\rangle+f_{u} U \cdot \bar{U}\right\} d x \leq \lambda^{u} \int_{\Omega} S U \cdot \bar{U} d x
$$

by Lemma 4.1 . Hence we obtain

$$
\int_{\Omega} \mathcal{A} U \cdot \bar{U} d x \leq \lambda^{u} \int_{\Omega} S U \cdot \bar{U} d x .
$$

Similarly, we have

$$
\int_{\Omega} \mathcal{B} \bar{V} \cdot V d x \leq \lambda^{v} \int_{\Omega} T \bar{V} \cdot V d x
$$


Since $\lambda^{u}<0$ and $\lambda^{v}<0$ if $(\varphi, \psi)$ is a nondegenerate mini-maximizer, there exists $\delta^{\prime}>0$ such that

$$
\int_{\Omega} \mathcal{A} U \cdot \bar{U} d x+\int_{\Omega} \mathcal{B} \bar{V} \cdot V d x<-\delta^{\prime}\left\{\int_{\Omega} S U \cdot \bar{U} d x+\int_{\Omega} T \bar{V} \cdot V d x\right\} .
$$

Then it follows from (4.11) that for some $\delta>0$, all eigenvalues satisfy $\Re\{\lambda\}<-\delta<0$. Thus the proof of Theorem 4.1 is completed.

Next, we consider the case where $u=\varphi$ is linearly unstable so that $\varphi$ is not a local minimizer of $E[u, \psi]$. (The case where $v=\psi$ is linearly unstable can be treated in the same manner.)

THEOREM 4.2. Let $(\varphi, \psi)$ be a solution of (4.2). Suppose that $u=\varphi$ is linearly unstable as a steady state of (4.3). Then for each $S$ fixed, if $\left\|T^{-1}\right\|$ is sufficiently small, $(u, v)=(\varphi, \psi)$ is linearly unstable as a steady state of (4.1).

Intuitively speaking, if $\left\|T^{-1}\right\|$ is sufficiently small, then $v$ is almost fixed to $\psi$ so that $u$ behaves like a solution of (4.3). Hence, if $u=\varphi$ is unstable, $(u, v)=(\varphi, \psi)$ is unstable if $\left\|T^{-1}\right\|$ is sufficiently small.

A remarkable property of mini-maximizers is that they must be spatially homogeneous if the domain $\Omega$ is convex.

THEOREM 4.3. Let $\Omega$ be a convex domain with $C^{3}$-boundary, and let $(\varphi, \psi)$ be a solution of (4.2). If $(\varphi, \psi)$ is spatially inhomogeneous, then $\lambda^{u}>0$ or $\lambda^{v}>0$.

The proof is based on the idea of Jimbo and Morita [3]. Similar results for minimizers were obtained by Casten and Holland [1] and Matano [7] for scalar reactiondiffusion equation, and by Jimbo and Morita [3] and Lopes [6] for gradient systems.

From this theorem, the following result is immediately obtained.

COROllary 4.3. Let $\Omega$ be a convex domain with $C^{3}$-boundary, and let $(\varphi, \psi)$ be a solution of (4.2). If $(\varphi, \psi)$ is spatially inhomogeneous, then $(u, v)=(\varphi, \psi)$ is linearly unstable as a steady state of (4.1) for some $S$ and $T$.

Let us apply the above results to the diffusive FitzHugh-Nagumo system

$$
\begin{cases}u_{t}=\Delta u+f(u)-v & \text { in } \Omega \\ \tau v_{t}=d \Delta v+\varepsilon(u-\gamma v) & \text { in } \Omega \\ \frac{\partial}{\partial \nu} u=0=\frac{\partial}{\partial \nu} v & \text { on } \partial \Omega\end{cases}
$$

where $\tau, d, \varepsilon>0$ and $\gamma \geq 0$ are positive parameters. Suppose that $\Omega$ is a convex domain with $C^{3}$-boundary and that $(u, v)=(\varphi, \psi)$ is a spatially inhomogeneous steady state of (4.12). The maximal eigenvalue of

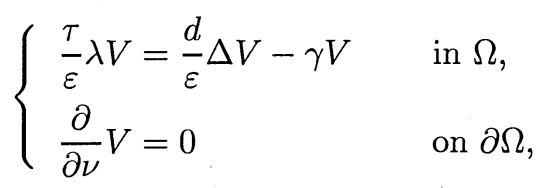

satisfies $\lambda^{v}=-\varepsilon \gamma / \tau<0$. Then, by Theorem 4.3, the maximal eigenvalue of

$$
\begin{cases}\lambda U=\Delta U+f_{u} U & \text { in } \Omega \\ \frac{\partial}{\partial \nu} U=0 & \text { on } \partial \Omega\end{cases}
$$


must satisfy $\lambda^{u}>0$. Thus, by virtue of Theorem 4.2, we obtain the following result.

THEOREM 4.4. Let $\Omega$ be a convex domain with $C^{3}$-boundary, and let $(u, v)=$ $(\varphi, \psi)$ be a steady state of (4.12). If $(\varphi, \psi)$ is spatially inhomogeneous, then there exists a constant $\tau_{*} \geq 0$ such that $(\varphi, \psi)$ is linearly unstable for all $\tau>\tau_{*}$.

Next, we consider the Gierer-Meinhardt system

$$
\begin{cases}u_{t}=\varepsilon^{2} \Delta u-u+\frac{u^{p}}{v^{q}}+\sigma & \text { in } \Omega, \\ \tau v_{t}=d \Delta v-v+\frac{u^{r}}{v^{s}} & \text { in } \Omega, \\ \frac{\partial}{\partial \nu} u=0=\frac{\partial}{\partial \nu} v & \text { on } \partial \Omega\end{cases}
$$

where the exponents are usually assumed to satisfy $p>1, q, r>0, s, \sigma \geq 0$ and

$$
\frac{p-1}{q}<\frac{r}{s+1}
$$

Suppose that $\Omega$ is a convex domain with $C^{3}$-boundary and that $(u, v)=(\varphi, \psi)$ is a spatially inhomogeneous positive steady state of (4.13). Since $s \geq 0$, the maximal eigenvalue of

$$
\begin{cases}q \tau \lambda V=q d \Delta V-q\left(1+s \varphi^{r} / \psi^{s+1}\right) V & \text { in } \Omega \\ \frac{\partial}{\partial \nu} V=0 & \text { on } \partial \Omega\end{cases}
$$

satisfies $\lambda^{v}<0$. Then, by Theorem 4.3, the maximal eigenvalue of

$$
\left\{\begin{array}{llrl}
r \lambda U & =r \varepsilon^{2} \Delta U+r\left(-1+p \varphi^{p-1} / \psi^{q}\right) U & & \text { in } \Omega, \\
\frac{\partial}{\partial \nu} U & =0 & & \text { on } \partial \Omega,
\end{array}\right.
$$

satisfies $\lambda^{u}>0$. Thus, by virtue of Theorem 4.2, we obtain the following result.

THEOREM 4.5. Let $\Omega$ be a convex domain with $C^{3}$-boundary, and let $(u, v)=$ $(\varphi, \psi)$ be a positive steady state of (4.13) with $p+1=r$ and $q+1=s$. If $(\varphi, \psi)$ is spatially inhomogeneous, then there exists a constant $\tau_{*} \geq 0$ such that the steady state is unstable for all $\tau>\tau_{*}$.

\section{REFERENCES}

[1] R. G. CASTEN AND C. J. Holland, Instability results for reaction diffusion equations with Neumann boundary conditions, J. Differential Equations, 27 (1978), pp. 266-273.

[2] J. W. Evans, Nerve axon equations: III. Stability of the nerve impulse, Indiana Univ. Math. J., 22 (1972), pp. 577-593.

[3] S. Jimbo AND Y. MORITA, Stability of nonconstant steady-state solutions to a Ginzburg-Landau equation in higher space dimensions, Nonlinear Analysis, 22 (1984), pp. 753-770.

[4] H. KIELhöFER, Stability and semilinear evolution equations in Hilbert space, Arch. Rational Mech. Anal., 57 (1974), pp. 150-165.

[5] M. KUWAMURA AND E. YANAGIDA, Instability criteria for a family of spatially periodic equilibira in gradient/skew-gradient systems, preprint.

[6] O. LoPES, Radial and nonradial minimizers for some radially symmetric functionals, Elec. J. Differential Equations, 1996 (1996), pp. 1-14. 
[7] H. Matano, Asymptotic behaviour and stability of solutions of semilinear elliptic equations, Publ. RIMS. Kyoto Univ., 15 (1979), pp. 401-454.

[8] I. TAKAGI, Stability of bifurcating solutions of the Gierer-Meinhardt system, Tohoku Math. J., 31 (1979), pp. 221-246.

[9] E. YANAGIDA, Standing pulse solutions in reaction-diffusion systems with skew-gradient structure, preprint.

[10] E. YANAGIDA, Mini-maximizers for reaction-diffusion systems with skew-gradient structure, preprint. 